\title{
Topological Insulators in Ternary Compounds with a Honeycomb Lattice
}

\author{
Hai-Jun Zhang ${ }^{1}$, Stanislav Chadov ${ }^{2}$, Lukas Müchler ${ }^{2}$, Binghai Yan ${ }^{1}$, \\ Xiao-Liang Qi $^{1}$, Jürgen Kübler ${ }^{3}$, Shou-Cheng Zhang ${ }^{1}$, Claudia Felser ${ }^{1,2}$ \\ ${ }^{1}$ Department of Physics, McCullough Building, Stanford University, Stanford, California 94305-404531 \\ ${ }^{2}$ Institut für Anorganische Chemie und Analytische Chemie, \\ Johannes Gutenberg - Universtität, 55099 Mainz, Germany,

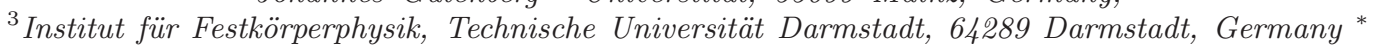

(Dated: October 8, 2018)

\begin{abstract}
One of the most exciting subjects in solid state physics is a single layer of graphite which exhibits a variety of unconventional novel properties. The key feature of its electronic structure are linear dispersive bands which cross in a single point at the Fermi energy. This so-called Dirac cone is closely related to the surface states of the recently discovered topological insulators. The ternary compounds, such as LiAuSe and $\mathrm{KHgSb}$ with a honeycomb structure of their Au-Se and $\mathrm{Hg}-\mathrm{Sb}$ layers feature band inversion very similar to $\mathrm{HgTe}$ which is a strong precondition for existence of the topological surface states. In contrast to graphene with two Dirac cones at $K$ and $K^{\prime}$ points, these materials exhibit the surface states formed by only a single Dirac cone at the $\Gamma$ point together with the small direct band gap opened by a strong spin-orbit coupling (SOC) in the bulk. These materials are centro-symmetric, therefore, it is possible to determine the parity of their wave functions, and hence, their topological character. Surprisingly, the compound KHgSb with the strong SOC is topologically trivial, whereas LiAuSe is found to be a topological non-trivial insulator.
\end{abstract}

PACS numbers: 71.20.-b,73.43.-f,73.20.-r

Keywords: spin Hall effect, topological insulators

The search for new materials with inverted band structure provides the basis for the Quantum Spin Hall effect (QSH) [1 13]. This new exciting field started with the prediction and experimental observation of the QSH in quantum wells in two-dimensional topological insulator of the binary semiconductor $\mathrm{HgTe}$ [1, 2]. In a series of single crystals, such as $\mathrm{Bi}_{2} \mathrm{Se}_{3}$, three-dimensional topological insulting behavior was observed in topological surface states appearing as Dirac cones [7-9]. Later on, the manifold of Heusler semiconductors with 18 valence electrons and a similar band inversion was proposed [10, 11]. Since this proposed class of materials is extremely rich, it provides much wider flexibility in design by tuning the band gap size and the spin-orbit coupling (SOC) magnitude. In addition, the multi-functionality allows the incorporation of new properties such as superconductivity or magnetism [10].

The structure of the XYZ Heusler compounds can be simply viewed as "stuffed" YZ-zinc-blende. Depending on the stuffing element $\mathrm{X}$ Heuslers are semiconducting or semi-metallic 14. Materials like ScPtBi are topologically similar to HgTe: the inversion of the conduction and valence bands occurs due to small electronegativity differences. Since $\mathrm{HgTe}$ and $\mathrm{ScPtBi}$ are both 2D topological insulators, the QSH is also expected in the corresponding quantum wells, as e.g. ScPtSb/ScPtBi, in full analogy to $\mathrm{CdTe} / \mathrm{HgTe}$. We emphasize that the check of parity at the time reversal points, as a sufficient condition for their topological character, is not possible here because of the absence of inversion symmetry [3] .

\footnotetext{
* felser@uni-mainz.de
}

Heusler compounds are similar to a stuffed diamond, correspondingly, it should be possible to find the "high Z" equivalent of graphene in a graphite-like structure with 18 valence electrons and with inverted bands. In this structure type with a lower symmetry compared to diamond three-dimensional topological behavior is realizable. Indeed, the honeycombs $\mathrm{KZnP}$ and $\mathrm{KHgSb}$, crystallizing in so-called $\mathrm{AlB}_{2}, \mathrm{Ni}_{2} \mathrm{In}$ or $\mathrm{ZrBeSi}$ structure types, exhibit band ordering similar to the cubic $\mathrm{CdTe}$ and $\mathrm{HgTe}$. However, in contrast to graphene, these compounds have a strong SOC which leads to a finite band gap at the $\Gamma$ point [15, 16]. The determination of the wave function parity at the time-reversal points for these materials [3] is now possible, since they are centrosymmetric.

In detail, $\mathrm{KHgSb}$, for instance, can be presented as a stuffed graphene in the following way: the electropositve $\mathrm{K}^{+}$is stuffed in a honeycomb lattice of $[\mathrm{HgSb}]^{-}$[17]. The $\mathrm{ZrBeSi}$ structure with additional stuffing electropositive main group elements is illustrated in Figure 1. One sees that the main group and the transition elements alternate within the same layer and between the layers, therefore the primitive unit cell consists of two formula units.

The number of ternary compounds crystallizing in this structure is nearly as large as in the Heusler family, however due to a weaker mean hybridization $\left(s p^{2}\right.$ versus $\left.s p^{3}\right)$ many of the 18 valence electron compounds exhibit substantially smaller gaps or are simply metallic. As we show in the following, a certain number of semiconducting materials of this family with ordinary and inverted band structures can be found. In contrast to $\mathrm{HgTe}$ and $\mathrm{ScPtBi}$, such materials with inverted structure exhibit real band gaps due to their higher structural anisotropy. For this reason the honeycomb compounds are three di- 
(a)

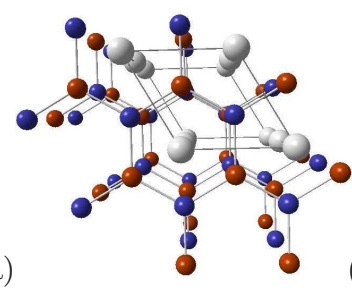

(b)

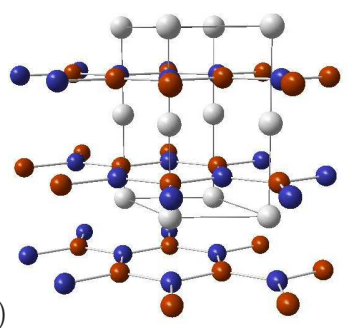

FIG. 1. (color online) Structure of KZnP, KHgSb, KCuSe and LiAuTe (ZrBeSi structure type) (a) shown in the $a-b$ plane and (b) along the $c$ axis. Thus, the ternary semiconductor (with 18 valence electrons) can be viewed as stuffed relative of graphite with the honeycomb lattice consisting of alternating late transition metals $(\mathrm{Ag}, \mathrm{Au}, \mathrm{Zn}, \mathrm{Hg})$ and main group elements (Se, Te, P, As, Sb).

mensional (3D), rather than 2D topological insulators. The 3D topological insulators exhibit an insulating energy gap in the bulk and gapless states on the surface protected by time-reversal symmetry [4]. These surface states exist only if there is an odd number of massless Dirac cones, with a single cone in the simplest case. The oddness is provided by the $Z_{2}$ topological invariant of the bulk [15, 18], thus any time-reversal invariant perturbation cannot open an insulating gap at the Dirac point on the surface.

The bulk band gap is defined by interplay of the lattice constant, spin-orbit coupling and the difference in electronegativities of the honeycomb sublattice constituents. In analogy to a ternary cubic semiconductors, new topological insulators can be found among the heavy $8 / 18$ valence electron relatives of graphene, i. e. in a graphite XYZ structure type. Suitable atomic combinations are: (i) $\mathrm{X}=\mathrm{Li}, \mathrm{Na}, \mathrm{K}, \mathrm{Rb}, \mathrm{Cs}$; $\mathrm{Y}=\mathrm{Zn}, \mathrm{Cd}, \mathrm{Hg}$ and $\mathrm{Z}=\mathrm{P}, \mathrm{As}$, $\mathrm{Sb}, \mathrm{Bi}$, or (ii) $\mathrm{X}=\mathrm{K}, \mathrm{Rb}, \mathrm{Cs}$; $\mathrm{Y}=\mathrm{Ag}, \mathrm{Au}$ and $\mathrm{Z}=\mathrm{Se}, \mathrm{Te}$, or (iii) $\mathrm{X}=$ rare earth, $\mathrm{Y}=\mathrm{Ni}, \mathrm{Pd}, \mathrm{Pt}$ and $\mathrm{Z}=\mathrm{P}, \mathrm{As}, \mathrm{Sb}$, $\mathrm{Bi}$. Some of these combinations are not yet synthesized, some crystallize in different forms such as variants of the $\mathrm{Cu}_{2} \mathrm{Sb}$ structure type (they will be the subject of another publication) and some, especially the rare earth containing compounds, are metallic. The known examples are KZnAs, KZnSb, KZnP, KHgAs, KHgSb, RbZnP, RbZnAs, RbZnSb, NaAuTe, KCuSe, KCuTe, KAuTe, and RbAuTe. Compounds such as LiAuSe, LiAuTe, CsAuTe, $\mathrm{KHgBi}$, and $\mathrm{CsHgBi}$ are likely to be synthesized and we consider them in the present study as well. As mentioned above, the electronic structure of these materials is similar to their cubic analogues. The alkaline ions $\mathrm{Li}^{+}, \mathrm{Na}^{+}$, $\mathrm{K}^{+}, \mathrm{Rb}^{+}$, and $\mathrm{Cs}^{+}$"stuff" the graphite type $\mathrm{YZ}$ planar sublattice. Since these 18-electron compounds form such closed-shell structures, they are all non-magnetic and semiconducting. The difference is that in the case of binary semiconductors or $\mathrm{C}_{1 b}$ Heuslers, the bonds within the YZ tetrahedrons are of $s p^{3}$ or $s d^{3}$ type, whereas in planar graphite-type layers the $\sigma$-type bonding occurs between the $s p^{2}$ or $s d^{2}$ orbitals. The remaining $p$ orbitals provide the $\pi$-type bonding interaction, similar to graphite.

The search for the topological character of the proposed materials is based on ab-initio calculation of the electronic structure (for details see the supplemental). An important peculiarity of hexagonal systems is emphasized here: their unit cell consists of two formula units which results in a doubling of the corresponding bands. More details are given in the supplemental (Fig. 1) where it is seen that for $\mathrm{KZnP}$ (KHgSb) at the $\Gamma$ point the doubled $s$-bands are split by about $10 \mathrm{meV}$. Thus for the weak coupling of the nearest honeycomb planes these doubled bands can become nearly indistinguishable. In the following we show that such doubling of the unit cell leads to a change of topology. Fortunately, the centro-symmetric space group (194) of the honeycomb-type compounds allows to make use of the parity eigenvalues [3, 8]. All relevant properties, i. e. the wave function parity in time-reversal symmetric $k$ points $(\Gamma(0,0,0), M(\pi, 0,0), L(\pi, 0, \pi), A(0,0, \pi))$, the $Z_{2}$ invariant, the average nuclear charge $\langle Z\rangle$, the band gap width $E_{\mathrm{g}}$ and corresponding lattice parameters are listed in Table I for the compounds studied here.

TABLE I. Wave function parities at the time-reversal points, $Z_{2}$ topological invariant [3], average nuclear charge $\langle Z\rangle$, band gap $E_{\mathrm{g}}$ and optimized lattice parameters.

\begin{tabular}{|c|c|c|c|c|c|c|c|c|c|}
\hline & $\Gamma$ & $M$ & $L$ & $A$ & $Z_{2}$ & $\langle Z\rangle$ & $E_{\mathrm{g}}[\mathrm{meV}]$ & $a[\mathrm{au}]$ & $c / a$ \\
\hline \hline $\mathrm{LiAgSe}$ & + & - & - & - & 1 & 28 & 1 & 8.507 & 1.512 \\
\hline LiAgTe & - & - & - & - & 0 & 34 & 45 & 8.979 & 1.526 \\
\hline LiAuSe & + & - & - & - & 1 & $38 .(6)$ & 50 & 8.353 & 1.670 \\
\hline LiAuTe & + & - & - & - & 1 & $44 .(6)$ & - & 8.818 & 1.663 \\
\hline $\mathrm{NaAgSe}$ & + & - & + & + & 1 & $30 .(6)$ & 10 & 8.544 & 1.737 \\
\hline $\mathrm{NaAgTe}$ & + & - & + & + & 1 & $36 .(6)$ & 3 & 9.031 & 1.719 \\
\hline $\mathrm{NaAuSe}$ & + & - & + & + & 1 & $41 .(3)$ & 15 & 8.427 & 1.855 \\
\hline $\mathrm{NaAuTe}$ & + & - & + & + & 1 & $47 .(3)$ & 30 & 8.894 & 1.823 \\
\hline $\mathrm{KAgSe}$ & - & - & + & + & 0 & $33 .(3)$ & 15 & 8.836 & 1.989 \\
\hline $\mathrm{KAgTe}$ & - & - & + & + & 0 & $39 .(3)$ & 230 & 9.193 & 1.958 \\
\hline $\mathrm{KAuSe}$ & - & - & + & + & 0 & 44 & 20 & 8.742 & 2.077 \\
\hline $\mathrm{KAuTe}$ & + & - & + & + & 1 & 50 & - & 8.780 & 2.097 \\
\hline \hline $\mathrm{LiZnAs}$ & - & - & - & - & 0 & 22 & 280 & 7.881 & 1.791 \\
\hline $\mathrm{LiZnSb}$ & - & - & - & - & 0 & 28 & 10 & 14.121 & 1.762 \\
\hline $\mathrm{LiHgAs}$ & + & - & - & - & 1 & $38 .(6)$ & - & 8.542 & 1.686 \\
\hline $\mathrm{LiHgSb}$ & + & - & - & - & 1 & $44 .(6)$ & - & 9.070 & 1.665 \\
\hline $\mathrm{NaZnAs}$ & - & - & + & + & 0 & $24 .(6)$ & 80 & 7.952 & 2.083 \\
\hline $\mathrm{NaZnSb}$ & - & - & + & + & 0 & $30 .(6)$ & 230 & 8.539 & 2.007 \\
\hline $\mathrm{NaHgAs}$ & - & - & + & + & 0 & $41 .(3)$ & - & 8.615 & 1.897 \\
\hline $\mathrm{NaHgSb}$ & - & - & + & + & 0 & $47 .(3)$ & 140 & 9.148 & 1.845 \\
\hline $\mathrm{KZnAs}$ & - & - & + & + & 0 & $27 .(3)$ & 160 & 7.993 & 2.419 \\
\hline $\mathrm{KZnSb}$ & - & - & + & + & 0 & $33 .(3)$ & 130 & 8.654 & 2.349 \\
\hline $\mathrm{KHgAs}$ & - & - & + & + & 0 & 44 & 80 & 8.515 & 2.214 \\
\hline $\mathrm{KHgSb}$ & - & - & + & + & 0 & 50 & 250 & 9.040 & 2.140 \\
\hline
\end{tabular}

From previous studies on binary and ternary cubic 


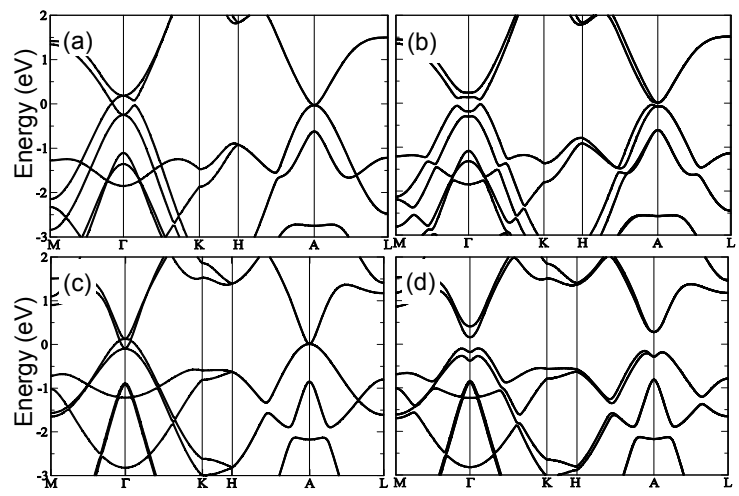

FIG. 2. Band structure of LiAuSe (a, b) and $\mathrm{KHgSb}(\mathrm{c}, \mathrm{d})$ calculated without $(\mathrm{a}, \mathrm{c})$ and with $(\mathrm{b}, \mathrm{d})$ SOC.

semiconductors [10] it follows that it is more probable to find the $Z_{2}=1$ topological insulator among heavier compounds (with stronger SOC). Indeed, their bands splitting scales roughly with the average nuclear charge $\langle Z\rangle=1 / N \sum_{i=1}^{N} Z_{i}$ where $N=2$ for binaries and $N=3$ for ternaries. This parameter sorts cubic systems almost along a straight line 10. However Table I clearly illustrates that this does not hold for the semiconductors of the $\mathrm{ZrBeSi}$ structure type. Indeed, only Lithium compounds within the $\mathrm{Zn}$ and $\mathrm{Hg}$ group show the expected trend. The compounds with $\mathrm{Zn}$ are topologically trivial whereas those with $\mathrm{Hg}$ are non-trivial insulators. Compounds with heavier alkaline metals $(\mathrm{Na}, \mathrm{K})$ are all trivial independently of whether or not they contain $\mathrm{Zn}$ or $\mathrm{Hg}$. Among the Ag- and Au-containing compounds more non-trivial systems are found, however the correlation between band inversion and $\langle Z\rangle$, as in the cubic semiconductors, is absent. For example, the topological insulator LiAgSe corresponds to $\langle Z\rangle=28$, whereas for the topologically trivial KHgSb system $\langle Z\rangle=50$.

In Figure2 the band structure of LiAuSe (upper panel) is compared with $\mathrm{KHgSb}$ (lower panel) calculated at time-reversal symmetric points with (right) and without (left) SOC. It follows that both compounds are semimetals with degeneracies at the $\Gamma$ and $A$ symmetry points if SOC is omitted. The degenerate $p_{\mathrm{x}}$ and $p_{\mathrm{y}}$ states mediate $\sigma$-type $\mathrm{Sb} / \mathrm{Se}$ and $\mathrm{Hg} / \mathrm{Au}$ bonding. The lower-lying $\mathrm{Hg} / \mathrm{Au}$ bands are of $s$ type, similar to $\mathrm{HgTe}$. Inclusion of SOC opens a band gap at the Fermi energy at the $\Gamma$ and $A$ points leading to the typical dips in the band structure for both compounds [19]. The resulting parities for both materials listed in Table I match only at the $M$ point, whereas at $\Gamma, A$, and $L$ they differ, leading to a trivial state in $\mathrm{KHgSb}$ and topological insulator in LiAuSe.

To understand the mechanisms of the band inversion and parity change in details one can track the band structure evolution near the $\Gamma$ point by starting from the simple atomic energy levels and subsequently introducing chemical bonding (step I), crystal field (II), and SOC (III). The resulting changes are schematically shown in Fig. 3. As an example we took the KZnP sys-
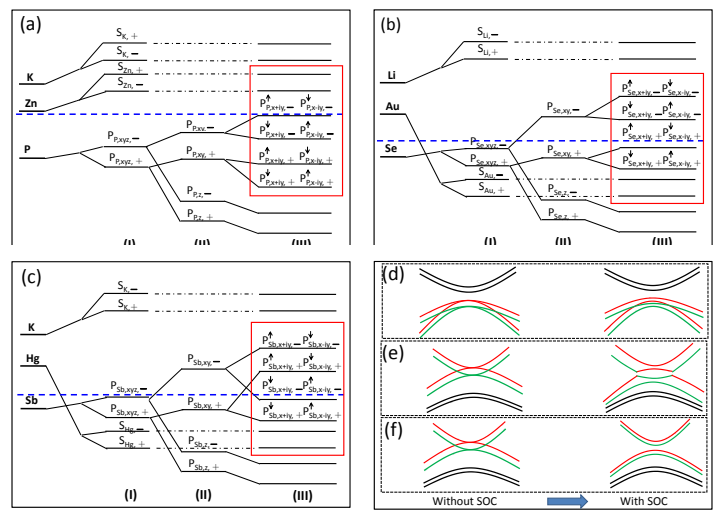

FIG. 3. (Color online) The structure of the atomic orbitals. (a) Evolution of atomic orbitals at the $\Gamma$ point in $\mathrm{KZnP}$ system, which has no inverted bands formed by $\mathrm{Zn} s$ and $\mathrm{P}$ $p_{\mathrm{x}, \mathrm{y}, \mathrm{z}}$-orbitals. Steps (I-III) represent the effect of turning on chemical bonding (I), crystal-field splitting (II) and SOC (III). The blue dashed line marks the Fermi energy. Similar scheme for LiAuSe (b) and KHgSb (c), (d), (e) and (f) represent the schematic band structure for KZnP, LiAuSe and KHgSb near the $\Gamma$ point, respectively. The black curves mark the orbitals with mainly $\mathrm{Zn}$ (or $\mathrm{Au}$ and $\mathrm{Hg}$ ) $s$-character, red and green curves are the rest of light and heavy hole-like bands.

tem. The low-lying $3 s$-orbital of $\mathrm{P}\left(3 s^{2} 3 p^{3}\right)$ can be neglected and the consideration can be restricted to the $s$-orbitals of $\mathrm{K}(4 s)$ and $\mathrm{Zn}\left(3 d^{10} 4 s^{2}\right)$ and the $p_{x, y, z}$ orbitals of $\mathrm{P}\left(3 s^{2} 3 p^{3}\right)$. In step I the chemical bonding is introduced. It is convenient to make use of the inversion symmetry and recombine the orbitals according to their parities. $\mathrm{K}$ and $\mathrm{Zn}$ give two even and two odd $s$ orbitals $\left|S_{\mathrm{K}, \pm}\right\rangle,\left|S_{\mathrm{Zn}, \pm}\right\rangle ; \mathrm{P}$ gives three odd and three even $p$-orbitals $\left|P_{\mathrm{P}, \mathrm{xyz}, \pm}\right\rangle$, where " \pm " are the parity labels. In step II the crystal field is switched on which splits the $\left|P_{\mathrm{P}, \mathrm{xy}, \pm}\right\rangle$ into $\left|P_{\mathrm{P}, \mathrm{xy}, \pm}\right\rangle$ and $\left|P_{\mathrm{P}, \mathrm{z}, \pm}\right\rangle$ according to the hexagonal symmetry. In step III the SOC is turned on. This leads to a splitting between $\left|P_{\mathrm{P}, \mathrm{x}+\mathrm{iy}, \pm, \downarrow(\uparrow)}\right\rangle$ and $\left|P_{\mathrm{P}, \mathrm{z}, \pm, \uparrow(\downarrow)}\right\rangle$ orbitals. The Fermi level falls into the middle of $\left|P_{\mathrm{P}, \mathrm{x}+\mathrm{iy},-, \downarrow}\right\rangle$ (or $\left.\left|P_{\mathrm{P}, \mathrm{x}-\mathrm{iy},-, \uparrow}\right\rangle\right)$ and $\left|P_{\mathrm{P}, \mathrm{x}+\mathrm{iy},+, \uparrow}\right\rangle$ (or $\left|P_{\mathrm{P}, \mathrm{x}-\mathrm{iy},+, \downarrow}\right\rangle$ ) as shown in Figure 3(a). No inversion occurs between the $s$-orbital of $\mathrm{K}$ (or Zn) and $p$-orbital of $\mathrm{P}$, similar to the CdTe case. However, this situation changes for the LiAuSe and $\mathrm{KHgSb}$ compounds. Due to the very delocalized character of $\mathrm{Au}(\mathrm{Hg}) d$-orbitals the $s$-orbital of $\mathrm{Li}(\mathrm{K})$ is pulled down below the $p$-orbital of $\mathrm{P}$, which leads to band inversion similar to the $\mathrm{HgTe}$ case [20]. The corresponding evolution for LiAuSe and $\mathrm{KHgSb}$ is shown in Figures 3 (b) and (c). In addition, $\left|P_{\mathrm{Sb}, \mathrm{x}+\mathrm{iy},-, \downarrow}\right\rangle$ (or $\left|P_{\mathrm{Sb}, \mathrm{x}-\mathrm{iy},-, \uparrow}\right\rangle$ ) and $\left|P_{\mathrm{Sb}, \mathrm{x}+\mathrm{iy},+, \uparrow}\right\rangle$ (or $\left.\left|P_{\mathrm{Sb}, \mathrm{x}-\mathrm{iy},+, \downarrow}\right\rangle\right)$ are inverted because of the strong SOC in $\mathrm{KHgSb}$. We emphasize that since there are two band inversions occurring in $\mathrm{KHgSb}$ it becomes topologically trivial. In contrast, LiAuSe exhibits inversion only between the $s$ - and $p$-orbitals since its SOC is too weak to invert $\left|P_{\mathrm{Sb}, \mathrm{x}+\mathrm{iy},-, \downarrow}\right\rangle\left(\right.$ or $\left.\left|P_{\mathrm{Sb}, \mathrm{x}-\mathrm{iy},-, \uparrow}\right\rangle\right)$ and $\left|P_{\mathrm{Sb}, \mathrm{x}+\mathrm{iy},+, \uparrow}\right\rangle$ (or $\left|P_{\mathrm{Sb}, \mathrm{x}-\mathrm{iy},+, \downarrow}\right\rangle$ ). Thus we conclude that LiAuSe is topologically non-trivial. The corresponding band struc- 


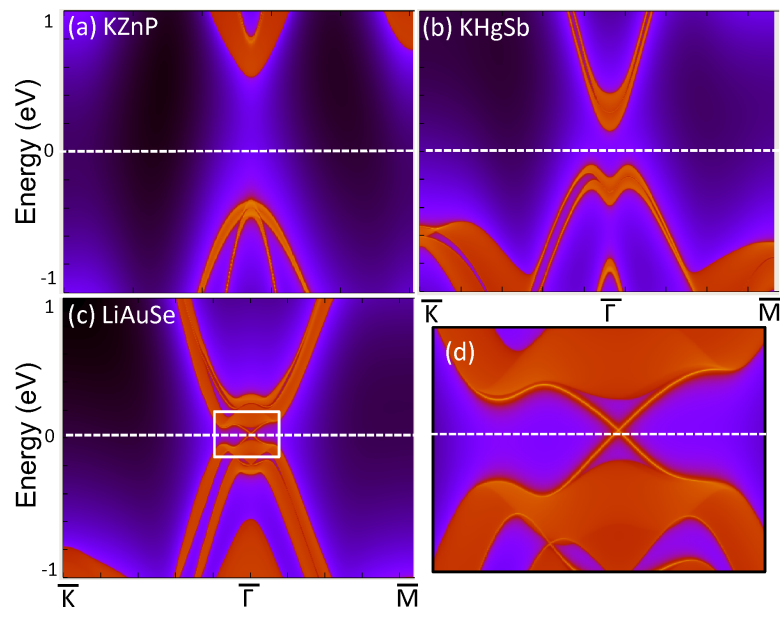

FIG. 4. (color online) The band structure of the semi-infinite (vacuum/solid-interface) corresponding to (a) trivial $\mathrm{KZnP}$, (b) non-trivial LiAuSe and (c) trivial KHgSb insulators, calculated along the $\bar{K}-\bar{\Gamma}-\bar{M}$ directions of the Brillouin zone. In contrast to $\mathrm{KZnP}$ and $\mathrm{KHgSb}$, LiAuSe exhibits the surface states seen in the bulk energy gap (zoomed in the inset (d)).

tures around the $\Gamma$ point for $\mathrm{KZnP}$, LiAuSe and $\mathrm{KHgSb}$ are shown schematically in Figures 3 (d-f). It is seen that in $\mathrm{KZnP}$ no band inversion occurs, in LiAuSe it occurs once and $\mathrm{KHgSb}$ twice.

For the final test of the topological/trivial character we directly calculate the surface states of the proposed systems. The electronic structure of the surface can be adequately described by the vacuum/solid interface within a unit cell which is sufficiently large along a certain direction. By utilizing the so-called decimation technique [21] such a unit cell can be extended to infinity which ensures correct boundary conditions. Such calculations typically require a formalism using fast decaying basis functions. In the present work we apply the Wannier functions approach [22]. As it follows from the band structures shown in Figure 4 only LiAuSe exhibits gapless surface states seen as a single Dirac cone within the bulk energy gap at the $\Gamma$ point. The estimate of its Fermi velocity gives about $1.8 \times 10^{5} \mathrm{~m} / \mathrm{s}$ which is smaller than that for $\mathrm{Bi}_{2} \mathrm{Se}_{3}$. In contrast, $\mathrm{KZnP}$ and $\mathrm{KHgSb}$ remain insulators at the surface. Thus the surface state calculation agrees with the bulk parity analysis and conclusively confirms the existence of the topologically non-trivial materials within proposed honeycomb type structure.

In conclusion we emphasize that topologically nontrivial systems can be found in the proposed class of honeycomb structure semiconductors. The interplay of mechanisms which are responsible for the topologically trivial or non-trivial character in these systems differs from the cubic semiconductors studied earlier. In particular it is shown that the strong SOC and weak inter-layer coupling causes a double inversion which in turn makes the compound trivial. In contrast to the topologically non-trivial cubic systems which exhibit a zero band gap in the bulk, the topologically non-trivial hexagonal materials provide the "natural" 3D topological materials with a real bulk band gap and gapless states at the surface.

\section{ACKNOWLEDGMENTS}

The work was supported by the supercomputing center at Stanford Institute Materials and Energy Science. The financial support of the DFG/ASPIMATT project (unit $1.2-\mathrm{A})$ is gratefully acknowledged.
[1] B. A. Bernevig, T. L. Hughes, and S. C. Zhang, Science 314, 1757 (2006).

[2] M. König, S. Wiedmann, C. Brüne, A. Roth, H. Buhmann, L. Molenkamp, X. L. Qi, and S. C. Zhang, Science 318, 766 (2007).

[3] L. Fu and C. L. Kane, Phys. Rev. B 76, 045302 (2007).

[4] L. Fu, C. L. Kane, and E. J. Mele, Phys. Rev. Letters 98, 106803 (2007).

[5] X. Dai, T. L. Hughes, X.-L. Qi, , Z. Fang, and S.-C. Zhang, Phys. Rev. B 77, 125319 (2008).

[6] D. Hsieh, D. Qian, L. Wray, Y. Xia, Y. S. Hor, R. J. Cava, and M. Z. Hasan, Nature 452, 970 (2008).

[7] Y. Xia, D. Qian, D. Hsieh, L. Wray, A. Pal, H. Lin, A. Bansil, D. Grauer, Y. S. Hor, R. J. Cava, and M. Z. Hasan, Nature Physics 5, 398 (2009).

[8] H. Zhang, C.-X. Liu, X.-L. Qi, X. Dai, Z. Fang, and S. C. Zhang, Nature Physics 5, 438 (2009).

[9] Y. L. Chen, J. G. Analyti, J. H. Chu, Z. K. Liu, S. K. Mu, X. L. Qi, H. J. Zhang, D. H. Lu, X. Dai, Z. Fang, S. C. Zhang, I. R. Fisher, Z. Hussain, and Z. X. Shen, Science 325, 178 (2009).

[10] S. Chadov, X.-L. Qi, J. Kübler, G. H. Fecher, C. Felser, and S.-C. Zhang, Nature Materials 9, 541 (2010).
[11] H. Lin, L. A. Wray, Y. Xia, S. Xu, S. Jia, R. J. Cava, A. Bansil, and M. Z. Hasan, Nature Materials 9, 546 (2010).

[12] X.-L. Qi and S.-C. Zhang, Physics Today 63, 33 (2010).

[13] J. Moore, Nature 464, 194 (2010).

[14] H. C. Kandpal, C. Felser, and R. Seshadri, J. Phys. D: Appl. Phys. 39, 776 (2006).

[15] C. L. Kane and E. J. Mele, Phys. Rev. Letters 95, 226801 (2005).

[16] B. A. Bernevig and S. C. Zhang, Phys. Rev. Letters 96, 106802 (2006).

[17] F. Casper, C. Felser, R. Seshardi, P. Sebastian, and R. Pöttgen, J. Phys. D: Appl. Phys. 41, 035002 (2008).

[18] C. L. Kane and E. J. Mele, Phys. Rev. Letters 95, 146802 (2005).

[19] M. Klintenberg, arXiv:1007.4838v1.

[20] A. Delin, Phys. Rev. B 65, 153205 (2002).

[21] H.-J. Zhang, C.-X. Liu, X.-L. Qi, X.-Y. Deng, X. Dai, S.C. Zhang, and Z. Fang, Phys. Rev. B 80, 085307 (2009).

[22] I. Souza, N. Marzari, and D. Vanderbilt, Phys. Rev. B 65, 035109 (2001). 


\title{
Supplementary material: Topological Insulators in Ternary Compounds with a Honeycomb Lattice
}

\author{
Hai-Jun Zhang ${ }^{1}$, Stanislav Chadov ${ }^{2}$, Lukas Müchler ${ }^{2}$, Binghai Yan ${ }^{1}$, \\ Xiao-Liang $\mathrm{Qi}^{1}$, Jürgen Kübler ${ }^{3}$, Shou-Cheng Zhang ${ }^{1}$, Claudia Felser ${ }^{1,2}$ \\ ${ }^{1}$ Department of Physics, McCullough Building, Stanford University, Stanford, California 94305-404531 \\ ${ }^{2}$ Institut für Anorganische Chemie und Analytische Chemie, \\ Johannes Gutenberg - Universtität, 55099 Mainz, Germany,

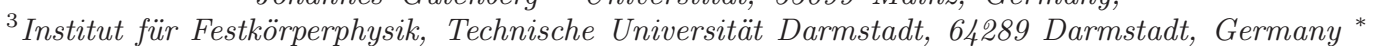

(Dated: October 8, 2018)

\begin{abstract}
This is supplementary material. We provide the details of electronic structure calculation, parity definition and illustrate the analogy in the band structures of cubic and hexagonal semiconductors.

PACS numbers: 71.20.-b,73.43.-f,73.20.-r

Keywords: spin Hall effect, topological insulators
\end{abstract}

Calculation of the electronic structure is performed by the plane-wave based pseudopotential BSTATE (Beijing Simulation Tool of Atomic TEchnology) package [1]. The exchange-correlation potential is treated within the Generalized Gradient Approximation (GGA) [2]. The lattice parameters listed in present work are obtained by doing the ab-initio geometry optimization.

The topological character is calculated by following Ref. [3]. The central quantity here is the parity product $\delta_{i}=\prod_{m=1}^{N} \xi_{2 m}\left(\Gamma_{i}\right)$, where $N$ is the number of filled bands and $\xi_{2 m}\left(\Gamma_{i}\right)$ is the parity eigenvalue of the $2 m$-th occupied band at the time reversal point $\Gamma_{i}$. The $Z_{2}$ invariant, $\nu_{0}=0$ or 1 , is then obtained from the product $(-1)^{\nu_{0}}=\prod_{i} \delta_{i}$.

Figure 1 illustrates the analogy between cubic $\mathrm{C}_{1 b}$ compounds and hexagonal semiconductors, by compar- ing the band structures of CdTe and HgTe with hexagonal ternaries $\mathrm{KZnP}$ and $\mathrm{KHgSb}$. Clear fingerprints can immediately be identified: both $\mathrm{CdTe}$ and $\mathrm{KZnP}$ exhibit a direct gap at the $\Gamma$ point formed by splitting of the conduction ( $s$-type) and the valence ( $p$-type) bands. By going from CdTe to $\mathrm{HgTe}$ and from $\mathrm{KZnP}$ to $\mathrm{KHgP}$ the bands at the $\Gamma$ point invert in a similar manner: the $s$ type shifts below the $p$-type band. However, in contrast to $\mathrm{HgTe}$ and other $\mathrm{C}_{1 b}$ Heuslers, the hexagonal symmetry with varying $c / a$ ratios allows to lift the degeneracy of the $p$-type bands and opens a finite band gap. As we mentioned this suggests KHgSb and the related materials as candidates for a 3D topological insulators, similar to $\mathrm{Bi}_{2} \mathrm{Se}_{3}$. It follows from the flat bands along the $K-H$ direction that the material is quasi two-dimensional which leads to a strong anisotropy of its transport properties.
[1] Z. Fang and K. Terakura, J. Phys.: Condens. Matter 14, 3001 (2002).

[2] J. P. Perdew, K. Burke, and M. Ernzerhof, Phys. Rev.
Letters 77, 3865 (1996).

[3] L. Fu and C. L. Kane, Phys. Rev. B 76, 045302 (2007).

* felser@uni-mainz.de 

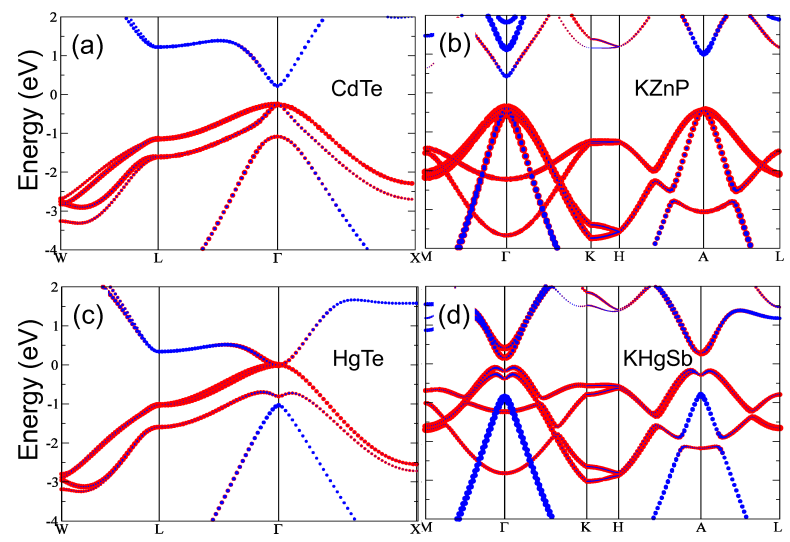

FIG. 1. (color online) Comparison of the band structures of cubic binaries (CdTe, HgTe) with hexagonal (KZnP, KHgSb) ternary compounds. In both $\mathrm{CdTe}$ and $\mathrm{KZnP}$ the valence and conduction bands are formed mainly by the $p$ (red) and the $s$ (blue) states, respectively. 